\title{
Influence of various immunosuppressive agents on the occurrence of endogenous bacteraemia in mice
}

\author{
Y. HIRAKATA* $\dagger$, N. FURUYA* $\dagger$, T. MATSUMOTO*, K. TATEDA* and K. YAMAGUCHI* \\ Department of Microbiology, Toho University School of Medicine, Tokyo 143 and $\dagger$ Department of Pulmonary \\ Medicine, Jichi Medical School, Tochigi 329-04, Japan
}

\begin{abstract}
Summary. The influence of six immunosuppressive agents on the occurrence of endogenous bacteraemia in mice was evaluated. The mortality rates in conventional ddY mice given cyclophosphamide (CY), fluorouracil (5-FU), methotrexate (MTX), cisplatin (CDDP) or FK-506 intraperitoneally, or dexamethasone (DXM) subcutaneously were 70, 100, 100, 100, 0 and $0 \%$, respectively. Pseudomonas aeruginosa was isolated from $70 \%$ of mice treated with CY but from only $10 \%$ of mice treated with $5-\mathrm{FU}$ and $30 \%$ treated with MTX. Enterobacteria were isolated from $90 \%$ of mice treated with 5-FU. Specific-pathogen-free (SPF) mice fed $P$. aeruginosa were also treated with these agents. All mice in the CY, 5-FU, MTX and CDDP groups died whereas mice treated with DXM and FK-506 showed $20 \%$ and $0 \%$ mortality, respectively. Pure cultures of $P$. aeruginosa were obtained from all of the mice treated with CY. Polymicrobial bacteraemia with $P$. aeruginosa and enterobacteria occurred in 5, 25, 5 and $5 \%$ of mice treated with 5-FU, MTX, CDDP and DXM, respectively. Enterobacterial bacteraemia was observed in $70 \%$ of mice treated with CDDP and in $5 \%$ of the DXM group. Different types of bacteraemia were induced by different immunosuppressive agents. The mechanism of immunosuppression may affect the frequency of bacteraemia and the causative organism.
\end{abstract}

\section{Introduction}

Bacteraemia in immunocompromised hosts frequently arises endogenously from the patient's own flora. ${ }^{1-3}$ Pseudomonas aeruginosa, Klebsiella pneumoniae and Candida albicans are typical causes of such infections, especially in bacteraemias originating in the intestinal flora. ${ }^{2-7}$ An animal model that resembles septicaemia in man is valuable for the evaluation of prophylaxis and treatment.

$P$. aeruginosa frequently causes endogenous systemic bacteraemia in leucopenic mice treated with cyclophosphamide $(\mathrm{CY})^{8}$ and bacteraemia with specific $P$. aeruginosa strains can be induced by treating mice fed the organisms with CY and ampicillin. ${ }^{9}$ These models have been used to investigate the pathogenic mechanism of endogenous bacteraemia, virulence factors and treatment. ${ }^{9-12}$ Escherichia coli, other enterobacteria and enterococci often cause portal bacteraemia only and are not isolated from cardiac blood. ${ }^{8,13}$

In many animal studies, $\mathrm{CY}$ is used to induce a compromised state. ${ }^{8-12,14-18}$ It is not well known

Received 10 May 1994; accepted 18 Aug. 1994. whether other immunosuppressive agents induce bacteraemia or other infections in animals, nor is it clear if $P$. aeruginosa bacteraemia can be induced by other immunosuppressive agents.

Six immunosuppressive agents were administered to conventional mice and specific-pathogen-free (SPF) mice fed $P$. aeruginosa strain D4 to compare mortality rates, frequency of occurrence of bacteraemia and causative organisms.

\section{Materials and methods}

\section{Reagents}

Sodium ampicillin (Viccillin; Meiji Seika Kaisha Ltd, Tokyo, Japan), cyclophosphamide (CY) (Endoxan; Shionogi and Co. Ltd, Osaka, Japan), fluorouracil (5-FU) (Kyowa Co. Ltd, Tokyo), cisplatin (CDDP) (Randa Inj.; Japan Chemical Co. Ltd, Tokyo), and dexamethasone (DXM) (Wako Pure Chemical Industries, Tokyo) were purchased from commercial sources. Methotrexate (MTX) and FK506 were kindly provided by Lederle Japan Co. Ltd, Tokyo, and Fujisawa Pharmaceutical Co. Ltd, Tokyo respectively. 


\section{Mice}

Conventional and SPF male ddY mice (Japan S.L.C. Co. Ltd, Shizuoka, Japan) weighing 20-24 g were used in the experiments. Mice were fed a sterile diet and were given sterile distilled water except during the period of oral administration of the bacteria.

\section{Bacterial strain and media}

$P$. aeruginosa strain D4 was isolated from cardiac blood of a mouse with systemic bacteraemia induced by CY as described previously. ${ }^{8}$ The organism was grown on Trypticase Soy Agar (BBL Microbiology Systems, Cockeysville, MD, USA) at $37^{\circ} \mathrm{C}$ for $18 \mathrm{~h}$. Bacteria were suspended in sterile saline $0.45 \%$ and adjusted to $10^{7} \mathrm{cfu} / \mathrm{ml}$ by spectrophotometry (UVIDEC-40; Jasco, Tokyo).

\section{Determination of endogenous bacteraemia}

Normal conventional mice. Endogenous bacteraemia was induced as described previously, ${ }^{8}$ with some modifications. Groups of 10 mice received ampicillin $200 \mathrm{mg} / \mathrm{kg} /$ day intraperitoneally for 15 days. Simultaneously, immunosuppressive agents were administered as follows: CY, 5-FU or MTX $200 \mathrm{mg} / \mathrm{kg} /$ day; FK-506 $100 \mathrm{mg} / \mathrm{kg} /$ day; CDDP $20 \mathrm{mg} / \mathrm{kg}$ every other day. DXM $10 \mathrm{mg} / \mathrm{kg}$ was given subcutaneously every other day.

Mice were observed four times each day and the number of deaths was recorded up to day 15. Cultures from cardiac blood were performed immediately after death. Survivors were killed by cervical dislocation and cultures were done at the end of the experiments. All samples were cultured immediately aerobically on sheep blood $5 \%$ agar (Nissui Pharmaceutical Co. Ltd, Tokyo) and trypticase soy agar at $37^{\circ} \mathrm{C}$ in humidified air for $24 \mathrm{~h}$. The isolates were identified by Vitek Identification Cards (Vitek Systems, Inc., Hazelwood, MO, USA).

$S P F$ mice fed $P$. aeruginosa D4. Faecal specimens from SPF mice were obtained before the study and examined to confirm the absence of $P$. aeruginosa. Mice were fed $P$. aeruginosa D4 in drinking water for 4 days and also received ampicillin $200 \mathrm{mg} / \mathrm{kg} /$ day during this period to disrupt the balance of the normal gastro-intestinal flora. Each immunosuppressive agent was administered on days 5, 7,9 and 11 as described above. Mice were monitored until day 15 and cultures of samples and identification of bacterial isolates were performed as described above.

\section{Results}

Survival of mice treated with immunosuppressive agents

Conventional mice. Mice treated with $\mathrm{CY}$ began to die on day 6 and their final mortality rate was $70 \%$. Mice treated with CDDP also started to die on day 6 and by day 8 all had died. Mice treated with 5-FU or MTX began to die later, but all mice died within the 15 days. In contrast, none of the mice treated with DXM or FK-506 died (fig. 1).

$S P F$ mice fed $P$. aeruginosa D4. Mice treated with

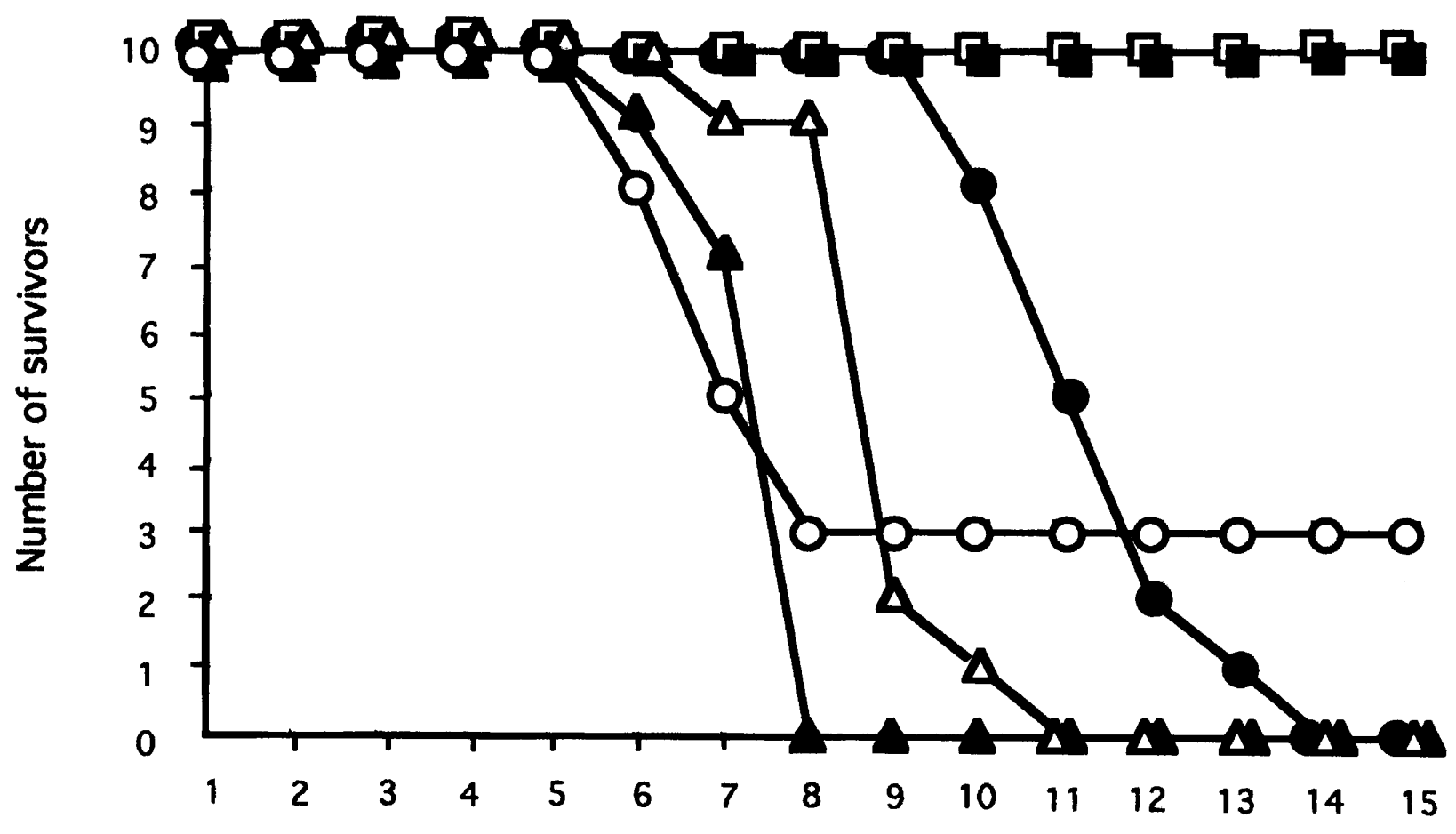

Day no.

Fig. 1. Survival of groups of 10 conventional mice treated with immunosuppressive agents: $O, C Y ; \mathbf{0}, 5-F U ; \triangle$, MTX; $\mathbf{\Delta}, \mathrm{CDDP} ; \square$, DXM; $\mathbf{\square}$, FK-506. 


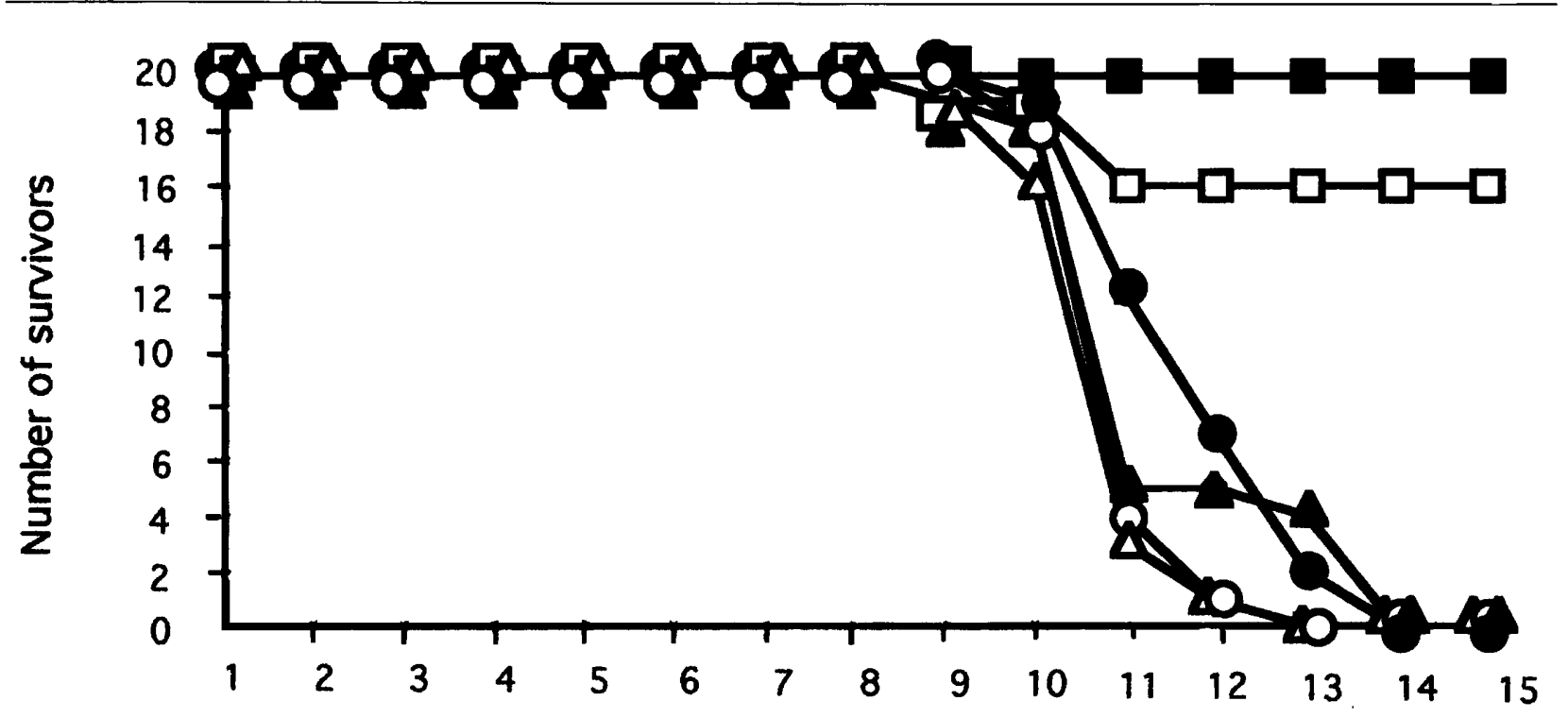

Day no.

Fig. 2. Survival of groups of $20 \mathrm{SPF}$ mice fed $P$. aeruginosa $\mathrm{D} 4$ and treated with immunosuppressive agents: $O, \mathrm{CY} ; \boldsymbol{\bullet}, 5$-FU; $\triangle$, MTX; $\boldsymbol{A}$, CDDP; $\square$, DXM; $\boldsymbol{\square}$, FK-506.

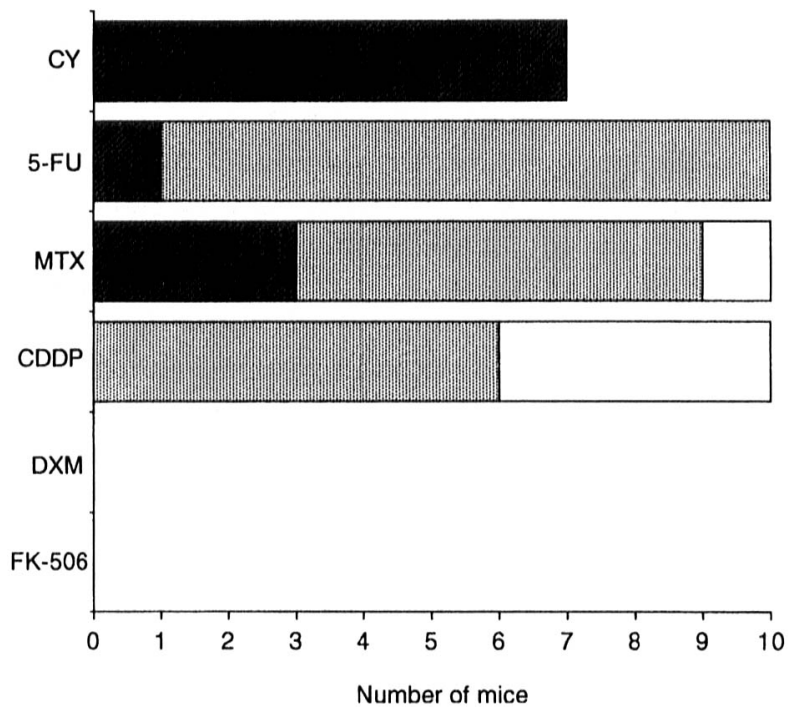

Fig. 3. Organisms isolated from cardiac blood of conventional mice treated with ampicillin and immunosuppressive agents and dying

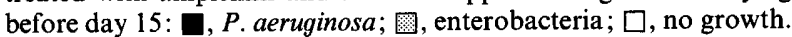

Table I. Enterobacteria isolated from cardiac blood of conventional mice treated with immunosuppressive agents

\begin{tabular}{lccc}
\hline & \multicolumn{3}{c}{ Number of mice treated with } \\
\cline { 2 - 4 } Species & 5-FU & MTX & CDDP \\
\hline K. pneumoniae & 4 & 0 & 0 \\
K. oxytoca & 3 & 3 & 1 \\
Enterobacter cloacae & 2 & 1 & 0 \\
$M$. morganii & 0 & 1 & 4 \\
$M$. morganii and $K$. oxytoca & 0 & 1 & 1 \\
\hline
\end{tabular}

CY died between days 9 and 13. Mice treated with 5FU, MTX or CDDP also showed a similar pattern of mortality, whereas most of the mice treated with DXM or FK-506 survived (fig. 2).
Table II. Enterobacteria isolated together with $P$. aeruginosa from cardiac blood of SPF mice treated with immunosuppressive agents

\begin{tabular}{lcccc}
\hline & \multicolumn{4}{c}{ Number of mice treated with } \\
\cline { 2 - 5 } Species & 5-FU & MTX & CDDP & DXM \\
& 1 & 1 & 0 & 0 \\
\hline K. pneumoniae & 1 & 0 & 1 & 0 \\
K. oxytoca & 0 & 5 & 0 & 1 \\
$P r$. mirabilis & 0 & 1 & 0 & 0 \\
$M$. morganii and Pr. mirabilis & & & & \\
\hline
\end{tabular}

Table III. Enterobacteria isolated without $P$. aeruginosa from cardiac blood of SPF mice treated with immunosuppressive agents

\begin{tabular}{lcc}
\hline & \multicolumn{2}{c}{ Number of mice treated with } \\
\cline { 2 - 3 } Species & CDDP & DXM \\
\hline E. coli & 1 & 0 \\
M. morganii & 8 & 0 \\
Pr. mirabilis & 5 & 1 \\
\hline
\end{tabular}

\section{Organism isolated from cardiac blood}

Conventional mice. $P$. aeruginosa strains were isolated from seven, one and three mice treated with $\mathrm{CY}$, 5-FU and MTX, respectively. Enterobacteria were isolated from nine, six and six mice treated with 5-FU, MTX, CDDP (fig. 3). Table I shows the species of enterobacteria isolated from these mice. Klebsiella spp. were the commonest cause of endogenous bacteraemia in mice treated with 5-FU or MTX. No bacteria were isolated from $10 \%$ of mice in the MTX group nor from $40 \%$ treated with CDDP. Amongst mice killed after 15 days, Morganella morganii was isolated from 


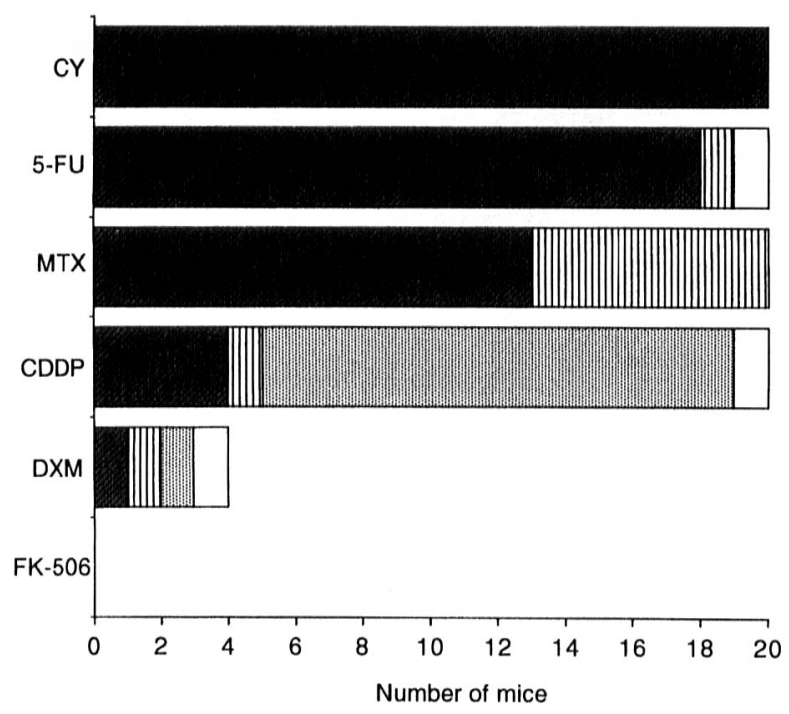

Fig. 4. Organisms isolated from cardiac blood of SPF mice fed $P$ aeruginosa D4 and treated with ampicillin and immunosuppressive agents and dying before day $15: \square, P$. aeruginosa $;, P$. aeruginosa and enterobacteria; 图, enterobacteria; $\square$, no growth.

two out of three mice treated with CY but cardiac blood from mice treated with DXM or FK-506 was sterile.

SPF mice fed $P$. aeruginosa D4. Pure cultures of $P$. aeruginosa were obtained from 100, 90, 65, 20 and $5 \%$ of mice treated with CY, 5-FU, MTX, CDDP and DXM, respectively. Polymicrobial bacteraemia ( $P$. aeruginosa and enterobacteria) occurred in 5, 25, 5 and $5 \%$ of mice treated with 5-FU, MTX, CDDP and DXM. The species isolated most frequently from mice treated with MTX was Proteus mirabilis (table II). Enterobacterial bacteraemia without $P$. aeruginosa was observed in 70 and $5 \%$ of mice treated with CDDP and DXM (table III). $M$. morganii and Pr. mirabilis were isolated more frequently from CDDP. treated mice. Only one mouse each in the groups treated with 5-FU, CDDP and DXM and dying before day 15 remained free of bacteraemia (fig. 4).

\section{Discussion}

$K$. pneumoniae, $E$. coli and $P$. aeruginosa are frequently involved in nosocomial septicaemia. ${ }^{3,19}$ Several outbreaks of infections including septicaemia by multi-resistant $K$. pneumoniae strains have been reported. 5,20,21 Epidemiological investigations have revealed that the reservoir for $K$. pneumoniae is the patients gastro-intestinal tract. ${ }^{5,6}$

We have reported previously that CY often induces bacteraemia due to endogenous $P$. aeruginosa in mice. ${ }^{8}$ Moreover, bacteraemia with specific $P$. aeruginosa strains was induced by administering CY and ampicillin to mice fed the organism. ${ }^{9}$ These animal models are useful for the investigation of the pathophysiology of endogenous bacteraemia, virulence factors of $P$. aeruginosa, and therapeutic interventions. ${ }^{9,10-12}$ Collins et $a .^{14}$ and Opal et al. ${ }^{15}$ described a model of endogenous $P$. aeruginosa bacteraemia in rats for the evaluation of antibiotic treatments and immunotherapy. Unlike most models of infection, which employ intraperitoneal or intravenous routes of infection, these models incorporated other steps, such as bacterial colonisation, overgrowth and invasion. Consequently, they closely mimic the pathophysiology of septicaemia in man. ${ }^{9,11,14}$

Enterobacteria such as $K$. pneumoniae and $E$. coli often cause only portal bacteraemia in CY-treated mice because they are easily cleared from blood by the reticulo-endothelial system, especially by Kupffer cells in the liver. ${ }^{8,13}$ Therefore, suitable immunosuppressive agents other than CY should be used to establish a model of endogenous bacteraemia due to enterobacteria. Braude et $a l^{22}$ and Ziegler et al. ${ }^{23}$ induced endogenous bacteraemia by feeding $E$. coli and $K$. pneumoniae to coliform-free rabbits conditioned with nitrogen mustard and inserting a temperature probe into the rectum. These animal models resemble the overwhelming septic shock observed after pelvic instrumentation. However, it is difficult to perform experiments with sufficient numbers of animals because of the size and cost of rabbits.

In this study, significant differences in mortality rates, frequency of bacteraemia and causative organisms were observed among mice treated with various immunosuppressive agents. CY frequently induced $P$. aeruginosa bacteraemia in both conventional mice and SPF mice fed $P$. aeruginosa D4. In contrast, CDDP often caused enterobacterial bacteraemia but mice treated with CDDP frequently died without bacteraemia, suggesting that the dose employed killed mice by acute toxicity before bacteraemia developed. DXM and FK-506 seldom induced bacteraemia. These immunosuppressive agents may not provide suitable models of endogenous bacteraemia. 5-FU induced different types of bacteraemia in conventional and SPF mice fed $P$. aeruginosa D4. Conventional mice frequently suffered septicaemia due to enterobacteria whereas SPF mice fed $P$. aeruginosa frequently suffered $P$. aeruginosa bacteraemia. This may be due to the difference in concentration of $P$. aeruginosa in the intestinal tracts of these mice. CY may induce $P$. aeruginosa bacteraemia selectively even when the concentration of $P$. aeruginosa is relatively low. The ability of MTX to induce bacteraemia was intermediate between CY and 5-FU.

The mechanisms of these differences are still to be clarified, but variation in target cells, degree of mucosal damage and the resident intestinal flora may account for the findings. The results may lead to the establishment of a new model of endogenous bacteraemia since immunosuppressive agents seem to be capable of selecting for certain bacteraemias. 


\section{References}

1. Bodey GP. Epidemiological studies of Pseudomonas species in patients with leukemia. Am J Med Sci 1970; 260: 82-89.

2. Schimpff SC, Young VM, Greene WH, Vermeulen GD, Moody MR, Wiernik PH. Origin of infection in acute nonlymphocytic leukemia. Significance of hospital acquisition of potential pathogens. Ann Intern Med 1972; 77: 707-714.

3. Young LS, Stevens P, Kaijser B. Gram-negative pathogens in septicaemic infections. Scand J Infect Dis 1982; 31 : 78-94.

4. Tancrede $\mathrm{CH}$, Andremont AO. Bacterial translocation and gram-negative bacteraemia in patients with hematological malignancies. J Infect Dis 1985; 152: 99-103.

5. De Champs C, Sauvant MP, Chanal C et al. Prospective survey of colonization and infection caused by expanded-spectrum- $\beta$-lactamase-producing members of the family $\mathrm{En}$ terobacteriaceae in an intensive care unit. J Clin Microbiol $1989 ; 27: 2887-2890$

6. Markowitz SM, Veazey JM, Macrina FL, Mayhall CG, Lamb VA. Sequential outbreaks of infection due to Klebsiella pneumoniae in a neonatal intensive care unit: implication of a conjugative R plasmid. $J$ Infect Dis $1980 ; 142$ : 106-112.

7. Richet $\mathrm{HM}$, Andremont $\mathrm{A}$, Tancrède $\mathrm{C}$, Pico JL, Jarvis WR. Risk factors for candidemia in patients with acute lymphocytic leukemia. Rev Infect Dis 1991; 13: 211-215.

8. Hirakata $\mathrm{Y}$, Tomono $\mathrm{K}$, Tateda $\mathrm{K}$ et al. Role of bacterial association with Kupffer cells in occurrence of endogenous systemic bacteraemia. Infect Immun 1991; 59: 289-294.

9. Hirakata Y, Kaku M, Tomono K et al. Efficacy of erythromycin lactobionate for treating Pseudomonas aeruginosa bacteraemia in mice. Antimicrob Agents Chemother $1992 ; 36$ : 1198-1203.

10. Hirakata Y, Furuya N, Tateda K, Kaku M, Yamaguchi K. In vivo production of exotoxin $\mathrm{A}$ and its role in endogenous Pseudomonas aeruginosa septicemia in mice. Infect Immun $1993 ; 61: 2468-2473$.

11. Furuya $\mathrm{N}$, Hirakata $\mathrm{Y}$, Tomono $\mathrm{K}$ et al. Mortality rates amongst mice with endogenous septicaemia caused by Pseudomonas aeruginosa isolates from various clinical sources. J Med Microbiol 1993; 39: 141-146.

12. Matsumoto T, Kaku M, Furuya $\mathrm{N}$ et al. Amphotericin Binduced resistance to Pseudomonas aeruginosa infection in mice. J Antibiot 1993; 46: 777-784.
13. Hirakata $\mathrm{Y}, \mathrm{Kaku} \mathrm{M}$, Furuya $\mathrm{N}$ et al. Effect of clearance of bacteria from the blood on the development of systemic bacteraemia in mice. J Med Microbiol 1993; 38: 337-344.

14. Collins HH, Cross AS, Dobek A, Opal SM, MaClain JB, Sadoff JC. Oral ciprofloxacin and a monoclonal antibody to lipopolysaccharide protect leukopenic rats from lethal infection with Pseudomonas aeruginosa. J Infect Dis 1989; 159: 1073-1082.

15. Opal SM, Cross AS, Kelly NM et al. Efficacy of a monoclonal antibody directed against tumor necrosis factor in protecting neutropenic rats from lethal infection with Pseudomonas aeruginosa. $J$ Infect Dis $1990 ; 161$ : 1148-1152.

16. Patterson TF, Fothergill AW, Rinaldi MG. Efficacy of itraconazole solution in a rabbit model of invasive aspergillosis. Antimicrob Agents Chemother 1993; 37: $2307-$ 2310.

17. Melissen PMB, van Vianen W, Bakker-Woudenberg IAJM. Treatment of Klebsiella pneumoniae septicemia in normal and leukopenic mice by liposome-encapsulated muramyl tripeptide phosphatidylethanolamide. Antimicrob Agents Chemother 1994; 38: 147-150.

18. Alder J, Mitten M, Jarvis K, Gupta P, Clement J. Efficacy of clarithromycin for treatment of experimental Lyme disease in vivo. Antimicrob Agents Chemother 1993; 37: 1329-1333.

19. Bone RC. Gram-negative sepsis: a dilemma of modern medicine. Clin Microbiol Rev 1993; 6: 57-68.

20. Brun-Buisson C, LeGrand P, Philippon A, Montravers F, Ansquer M, Duval J. Transferable enzymatic resistance to third-generation cephalosporins during nosocomial outbreak of multiresistant Klebsiella pneumoniae. Lancet 1987; 2: 302-306.

21. Sirot J, Chanal C, Petit A, Sirot D, Labia R, Getbaud G. Klebsiella pneumoniae and other enterobacteriaceae producing novel plasmid-mediated $\beta$-lactamases markedly active against third-generation cephalosporins: epidemiologic studies. Rev Infect Dis 1988; 10: 850-859.

22. Braude AI, Douglas H, Jones J. Experimental production of lethal Escherichia coli bacteraemia of pelvic origin. $J$ Bacteriol 1969; 98: 979-991.

23. Ziegler EJ, Douglas H, Sherman JE, Davis CE, Braude AI. Treatment of $E$. coli and klebsiella bacteraemia in agranulocytic animals with antiserum to a UDP-gal epimerasedeficient mutant. J Immunol 1973; 111: 433-438. 